\title{
Adult vitelliform macular degeneration: diagnosis and natural history*
}

\author{
GIL A. EPSTEIN AND MAURICE F. RABB \\ From the Aaron Fox Retino-Vascular Laboratory, Department of Ophthalmology, \\ Michael Reese Hopsital and Medical Center, Chicago, Illinois 60616, USA
}

SUMMARY True vitelliform dystrophy rarely appears in the adult population. We describe 10 cases in adults of bilateral vitelliform lesions clinically mistaken for Best's disease. Fluorescein angiography is a useful tool in distinguishing this dystrophy from Best's disease or other diseases. The angiographic findings suggest pigment epithelial disease. Adult vitelliform degeneration may lead to dry atrophic macular degeneration in a similar fashion as macular drusen. Symptoms and visual findings in these patients are fairly stable, and may be only slowly progressive in spite of ophthalmoscopic and fluorescein angiographic changes over a period of years. The electro-oculogram is useful in separating adult vitelliform macular degeneration from true vitelliform dystrophy.

For the past 70 years the 'poached egg' appearance of the macular lesion in vitelliform macular dystrophy has been described in various ways. The example presented by Best in 1905 has become the standard and since has been known as Best's disease. ${ }^{1}$ Other descriptive terms are vitelliform, ${ }^{2}$ vitelliruptive macular degeneration, ${ }^{3}$ vitelline macular degeneration, ${ }^{4}$ vitelliform dystrophy of the fovea, ${ }^{56}$ and inherited macular cyst. ${ }^{7}$

The classic presentation of the disease is a bilateral and symmetrical maculopathy with an autosomal dominant mode of inheritance. ${ }^{6} 8$ The vitelliform lesion typically measures $\frac{1}{2}$ to 3 discs diameters, and usually presents before the second decade of life. Visual acuity is frequently normal but may be minimally decreased in the vitelliform stage of Best's disease. A mild red-green dyschromatopsia is seen in later stages of the disease.

Fluorescein angiography typically shows hypofluorescence in the area corresponding to the vitelliform lesion in Best's disease. ${ }^{910}$ The electrooculogram (EOG) findings are pathologically subnormal. Deutman demonstrated subnormal EOGs in family members of patients with proved Best's disease, suggesting detection of a carrier state. ${ }^{56}$

Vitelliform macular dystrophy is believed to be a disease of retinal pigment epithelium. ${ }^{6} 8$ The intact

*This paper was presented at the Chicago Ophthalmological Society on 16 April 1979.

Correspondence to Dr Maurice F. Rabb, c/o Department of Ophthalmology, Michael Reese Hospital and Medical Center, 2929 South Ellis, Chicago, Illinois 60616, USA. vitelliform lesion ultimately leads to chorioretinal atrophy, with the terms 'scrambled egg' and 'pseudohypopyon' employed to describe stages in the natural progression of Best's disease.

Gass $^{11} 12$ described 9 cases of what he termed 'peculiar foveolar macular dystrophy'. His patients had bilateral, symmetrical, slightly raised yellow subretinal lesions that were $1 / 3$ disc diameter with a central pigment spot. The onset of the disease was primarily in the $\mathbf{3 0}$ to 50 year age group, with symptoms of blurred vision and metamorphopsia. There was a slow progression of visual loss in these patients. Gass found normal to slightly abnormal EOG readings in these cases. Fluorescein angiography showed either a hypofluorescent lesion or, more typically, a small ring of hyperfluorescence surrounding a hypofluorescent foveolar spot. Pathological changes were noted in the retinal pigment epithelium, and there was some question of the relationship of this entity to familial drusen.

In 1977 Fishman et al. described 3 patients with bilateral vitelliform-like lesions who had normal EOG findings. ${ }^{13}$ These authors used the term 'pseudovitelliform macular degeneration' to describe the condition. These patients were all older than 45 years of age and had a visual acuity of 20/100 or better. Fluorescein angiography showed hyperfluorescence surrounding the foveolar region, and they considered this was due to leakage from the perifoveolar capillaries. The authors emphasised the use of EOG in differentiating pseudovitelliform degeneration from Best's disease.

Kingham and Lochen described 6 cases of vitelli- 


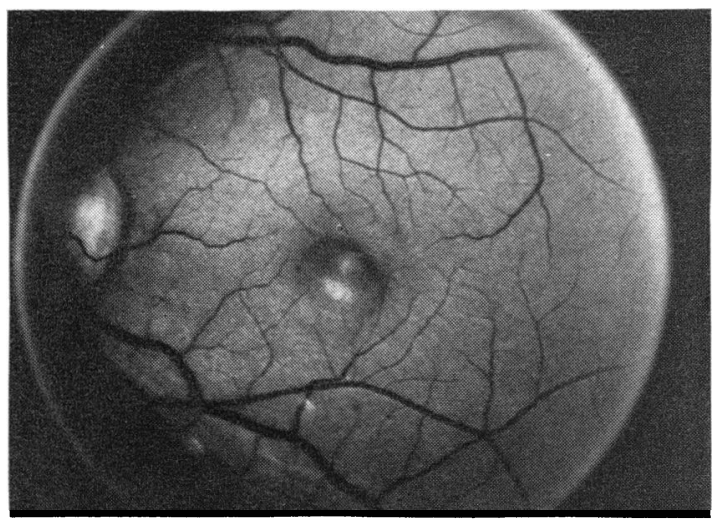

Fig. 1 (Case 1) Left macula with vitelliform-like lesion.

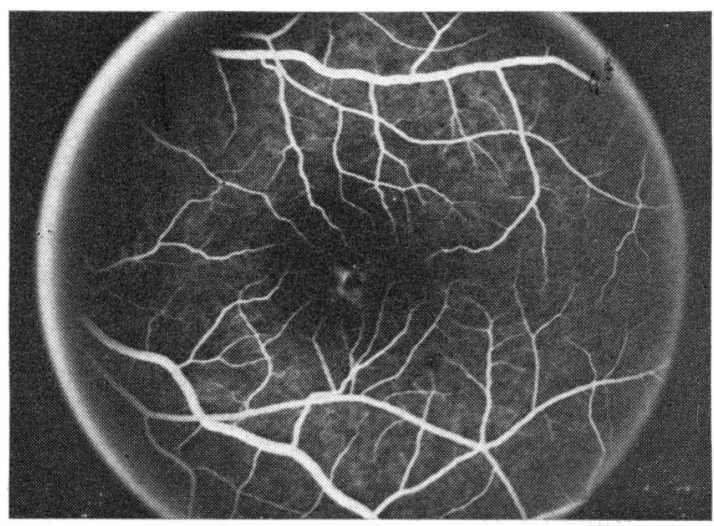

Fig. 2 (Case 1) Late venous phase angiogram of left eye showing ring hyperfluorescence with minimal subretinal leakage in macular region.

form macular degeneration with normal EOGs. ${ }^{14}$ Angiography in their cases revealed hyperfluorescence due to pigment epithelial changes. Leakage in the late stages was thought to arise from the choroid and not the perifoveal capillary network.

We investigated 10 cases of adult vitelliform lesions. When initially seen, the diagnosis of vitelliform macular dystrophy was considered. However, the age of presentation and the results of the retinal function tests ruled out Best's disease. Emphasis is directed to the diagnosis and natural history of adult vitelliform macular degeneration.

\section{Case reports}

\section{CASE 1}

A 45-year-old black female complained of a gradual progression of blurring vision and metamorphopsia in the left eye for 3 years. There was no history of previous medical or ocular disease or ocular trauma.
Physical examination revealed $20 / 30$ vision bilaterally with her present correction of a +0.50 sphere in each eye. However, a +1.25 sphere improved the acuity to $20 / 25+$ in the right eye and a +1.00 sphere +0.50 cylinder axis $90^{\circ}$ resulted in $20 / 25+$ vision in the left. The anterior segments, pupils, extraocular movements, and intraocular pressures were within normal limits. Amsler grid, Goldmann perimetry, and colour vision testing gave normal results. Fundus examination disclosed normal-appearing optic discs. However, in both macular regions there were $\frac{1}{2}$ disc diameter, slightly raised, vitelliform-like lesions (Fig. 1).

Fluorescein angiography showed early hyperfluorescence in the macular region in a ring-like fashion in a window defect pattern OU. Minimal subretinal leakage of dye was seen bilaterally in late stages of the angiogram (Fig. 2). The EOG was 2.1 in the right eye and 1.7 in the left eye. Examination of 6 family members failed to disclose any retinal or macular disease. Evaluation of the patient 1 year later revealed no change in ocular symptoms. The best visual acuity was $20 / 25+$ OU, but her refraction changed to +0.50 sphere in the right eye and +0.50 sphere +0.25 cylinder axis $90^{\circ}$ in the left. Amsler grid, colour vision tests, and visual fields were unchanged.

In both macular regions the vitelliform lesions had evolved into flat 'scrambled' lesions secondary to retinal pigment epithelial changes (Fig. 3). The fluorescein angiogram showed hyperfluorescence in a window defect pattern in both eyes.

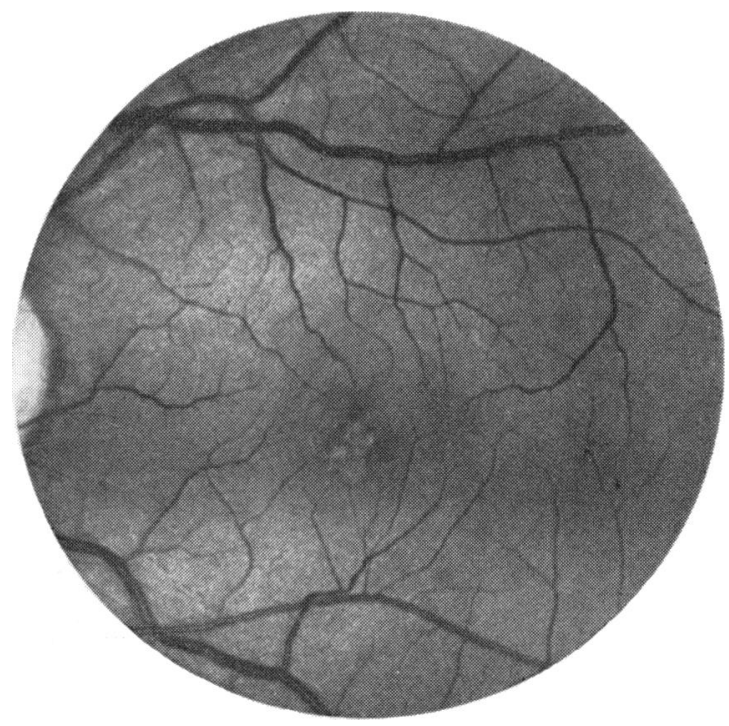

Fig. 3 (Case 1) Left macular region 1 year later. Notice scrambling of vitelliform lesion. 


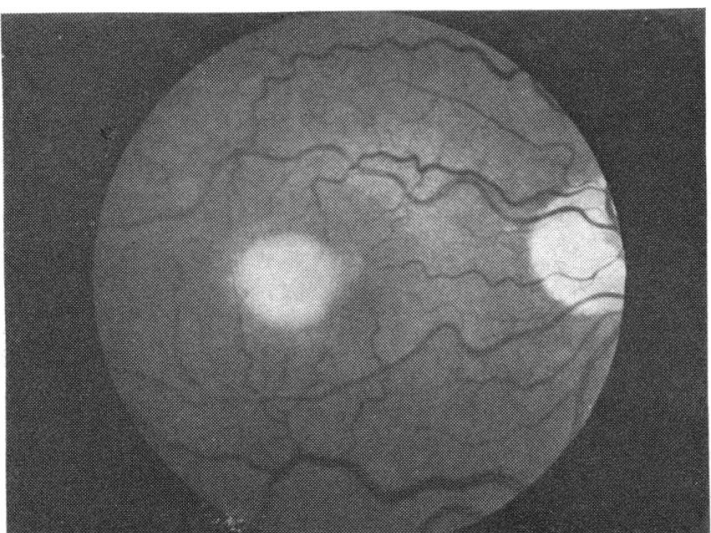

Fig. 4 (Case 2) Right macular region with $\frac{1}{2}$ to $3 / 4$ disc diameter raised adult vitelliform lesion (1974).

CASE 2

A 76-year-old white male complained of a gradual decrease of vision in both eyes, primarily at near vision, for the past 5 years. This patient was first described by Fishman et al. in $1977 .{ }^{13}$ The patient denied any history of metamorphopsia, photopsia, photophobia, or previous ocular problems. He had cardiac arrhthmias that were being treated with a pacemaker. Visual acuity was best corrected to 20/400 OU and Jaeger 10 in the right eye and Jaegar 8 in the left. There were $2+$ anterior cortical, and $2+$ nuclear sclerotic changes in both lenses, accounting for some loss of vision. Intraocular pressures, pupils, and extraocular movements were within normal limits. Amsler grid studies showed small areas of distortion in the inferotemporal quadrant in the right eye and in the superonasal quadrant in the left eye. Colour vision tested by isochromatic plates showed a red-green colour deficiency in both eyes. When the patient was seen in 1974, funduscopic examination revealed vitelliform-like lesions in both eyes that were approximately $\frac{1}{2}$ to $3 / 4$ disc diameters in size (Fig. 4). In addition, a 1 disc diameter pigmented naevus was noted superior to the left macula in 1974. Fluorescein angiography showed late subretinal leakage of dye in both eyes. An EOG in 1977 showed a value of 1.86 in the right eye and 2.08 in the left eye.

In 1978 ophthalmoscopic examination disclosed hyperpigmentation of the left foveal region at the level of the retinal pigment epithelium. Surrounding this was a ring of hypopigmentation. The naevus was unchanged. The right eye had an appearance of central areolar choroidal sclerosis (Fig. 5).

Angiographic study revealed ring-like hyperfluorescence in a window defect pattern surrounding central hypofluorescence in the left macular region.

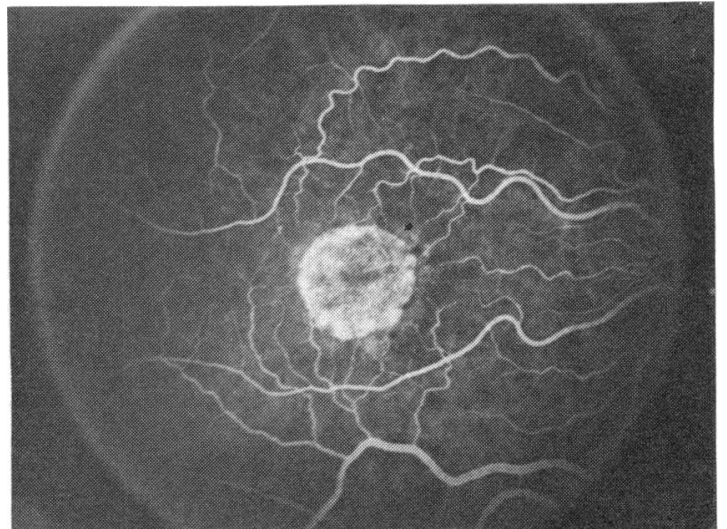

Fig. 5 (Case 2) Late venous angiogram right eye with central areolar choroidal sclerosis pattern (1978).

In the right eye early hyperfluorescence and a discrete circular pattern of central areolar choroidal sclerosis was noted (Fig. 4). There was no leakage or pooling of dye in either eye. Electrical studies were not permitted at this time in view of the patient's medical condition. Examination of the patient's 2 children failed to disclose any retinal or macular pathology.

\section{CASE 3}

A 70-year-old white female gave a 7- to 8-year history of progressive and gradual decrease of vision in both eyes. She denied other ocular symptoms, previous eye disease, or eye trauma. Pertinent medical history included hypertension medically controlled with a diuretic.

Ocular examination revealed a best corrected vision of 20/200 and 20/60 in the right and left eye, respectively. Near visions were Jaeger 8 in the right eye and Jaeger 3 in the left. The anterior segments were normal, except for $1+$ nuclear sclerotic changes in both' eyes. Applanation tonometry was within normal limits. Amsler grid showed superotemporal and inferonasal areas of distortion in the right eye. The Amsler grid in the left eye was within normal limits.

Fundus examination from July 1975 revealed a $1 / 3$ disc diameter vitelliform-like lesion that involved both macular regions. Fluorescein angiography in 1975 disclosed a ring of early hyperfluorescence in both macular regions. Late photographs demonstrated minimal subretinal leakage of dye.

Ophthalmoscopic examination in May 1978 revealed bilateral normal appearing optic discs. In the right macula a $\frac{1}{2}$ disc diameter vitelliform-like lesion was seen and appeared larger. A similar 1/3 disc diameter lesion with a central pigment spot was 


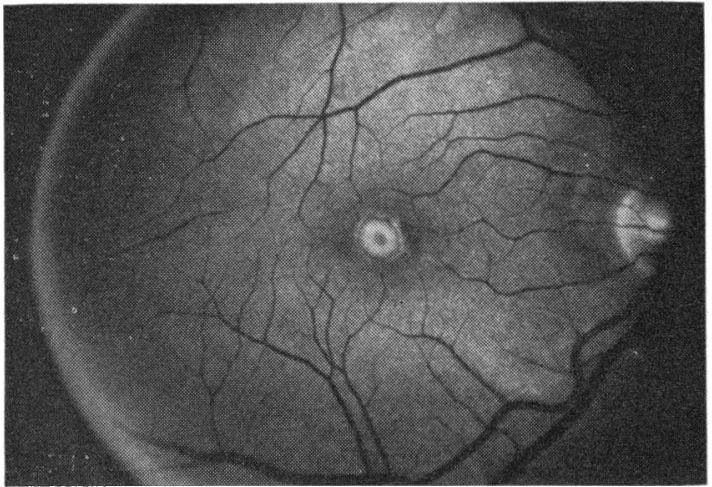

Fig. 6 (Case 4) Right eye showing umbilicated vitelliform lesion with central hyperpigmentation.

noted in the left eye. No other retinal or choroidal pathology was noted. Fluorescein angiography in 1978 showed an increase in the central hypofluorescence in the right macular region when compared with the 1975 study. However, a ring of early hyperfluorescence surrounding the fovea persisted, and late photographs showed minimal subretinal leakage of dye. Angiography of the left eye showed hyperfluorescence in the macular region in a window defect pattern with no leakage detected, consistent with dry atrophic macular degeneration. An EOG was not permitted. Examination of the patient's son did not disclose macular pathology.

\section{CASE 4}

A 63-year-old white male noted blurring of vision, and metamorphopsia in both eyes for the past 2 years. He denied previous medical or ocular problems and there was no history of ocular trauma. He was referred to our fluorescein angiography laboratory in August 1977, and visual acuity was 20/30 OU at that time. Ophthalmoscopically, $\frac{1}{4}$ disc diameter umbilicated vitelliform-like lesions were noted in both eyes (Fig. 6). Angiography revealed early hyperfluorescence in a ring-like configuration, which persisted as a window defect.

Examination in 1978 showed a best corrected visual acuity of $20 / 30+$ in both eyes. The anterior segments, extraocular movements, pupils, and intraocular pressures were within normal limits. Amsler grid testing revealed distortion of the central 4 squares in both eyes. Colour vision and visual field testing failed to disclose any abnormalities. The ophthalmoscopic and fluorescein examination had not changed from the study done 1 year previously. Examination of 2 family members did not reveal macular disease. Electrical studies were not permitted.

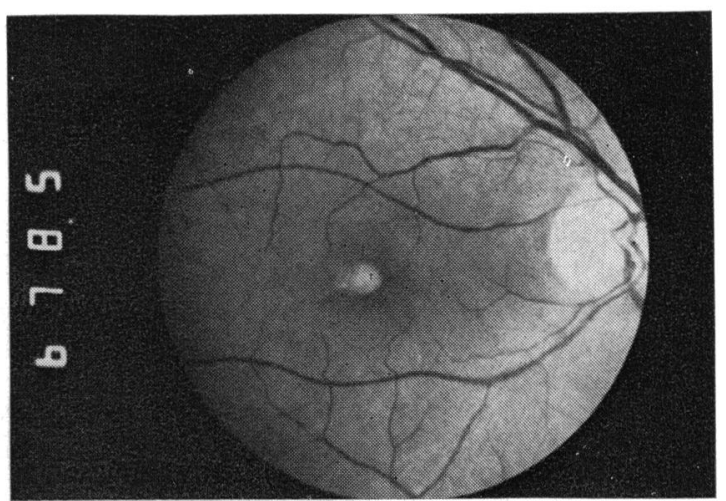

Fig. 7 (Case 6) Right eye with small raised vitelliform lesion.

\section{CASE 5}

A 78-year-old white female gave a 6-month history of blurred vision in both eyes. She denied other ocular symptoms or previous eye disease. Best corrected visual acuity was $20 / 50$ bilaterally. The external and anterior segment examinations of both eyes were normal. Ophthalmoscopy disclosed normal optic disc, but $1 / 3$ disc diameter vitelliform-like lesions were noted in both macular regions. Perimacular drusen were seen bilaterally. Fluorescein angiography showed minimal late leakage of dye beneath the sensory retina in both macular regions, more noticeable in the left eye. The EOG was 2.22 in the right eye and 2.40 in the left.

\section{CASE 6}

A 70-year-old white female had a 6-month history blurred vision in both eyes. She denied other ocular symptoms or previous eye disease. Medical problems were denied. Best corrected visual acuity was $20 / 30$ in the right eye and 20/40 in the left eye. Anterior segments, pupils, extraocular movements, and intraocular pressures were unremarkable bilaterally.

Funduscopic examination revealed vitelliformlike lesion in both macular regions (Fig. 7). No evidence of peripheral retinal disease was noted. Fluorescein angiography showed early hyperfluorescence in the macular regions which persisted as a window defect suggestive of retinal pigment epithelial changes. The EOG was within normal limits bilaterally (2.19 OD, $2.58 \mathrm{OS})$.

\section{CASE 7}

A 43-year-old white female described blurred vision in both eyes since 1961. She was given glasses at that time, with resultant visual improvement. In 1974 she had a decline in vision with metamorphop- 


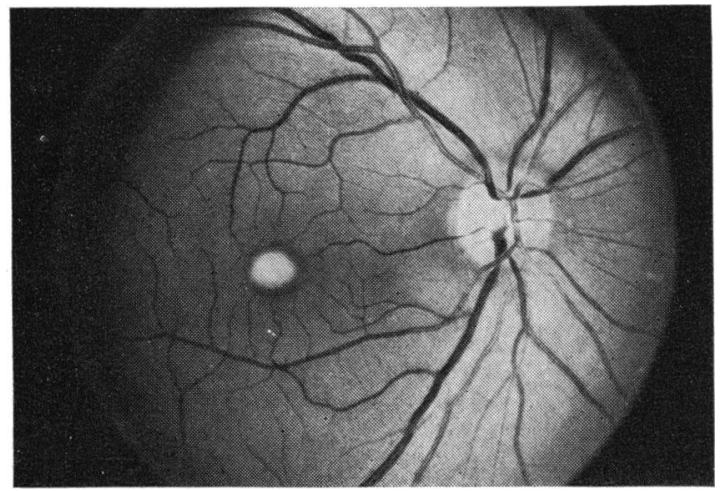

Fig. 8 (Case 7) Right eye with $\frac{1}{4}$ to $1 / 3$ raised adult vitelliform lesion (1974).

sia in both eyes, the right eye being worse than the left. Best corrected visual acuity at that time was $20 / 40$ in the right eye and $20 / 25$ in the left eye. Ophthalmoscopic examination in 1974 revealed bilateral macular vitelliform-like lesions (Fig. 8). Angiography revealed a faint ring of hyperfluorescence in the foveal region. In 1975 electrical studies were normal, and the patient was told that she did not have Best's disease.

In 1977 she had recurrent, blurred vision. Examination at another institution revealed vision acuities of 20/50 in the right eye with a +2.00 sphere and $20 / 30$ in the left eye with a +1.75 sphere. The remainder of the ocular examination was within normal limits. Electro-oculograms at that time revealed a light peak to dark trough ratio of 1.37 in the right eye and 1.66 in the left eye. On the basis of the EOG findings her condition was diagnosed as hereditary vitelliform macular degeneration.

In March 1978 the patient had a persistent

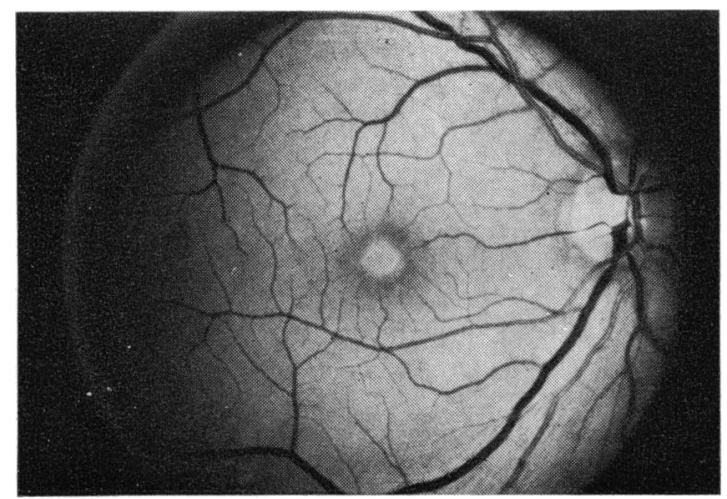

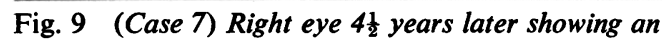
increase in central hyperpigmentation of the macular lesion (1978). decrease in visual acuity with metamorphopsia, primarily in the right eye. There were no medical problems or any use of medications. Her mother and sister also had a history of ocular problems. The patient's visual acuity was correctable to 20/40, Jaeger 3 with a +2.75 sphere +0.50 cylinder axis $70^{\circ}$ in the right eye, and 20/30, Jaeger 1 with a +1.75 sphere in the left. The Amsler grid showed distortion of the central 4 squares in the right eye. However, no visual field defect could be detected by Goldmann perimetry. Amsler grid and visual fields were normal in the left eye. Colour vision studies were normal bilaterally.

Ophthalmoscopically, bilateral normal optic discs were seen. However, in each macular region there was an approximate $1 / 3$ disc diameter vitelliformlike lesion. In the right foveolar region there was a small focal area of central hyperpigmentation at the level of the retinal pigment epithelium that was not seen ophthalmoscopically in 1974 (Fig. 9).

Fluorescein angiography revealed more hyperfluorescence, which persisted as a window-defect pattern in both eyes when compared with the 1974 angiogram (Fig. 10). There was no evidence of leakage or pooling of fluorescein. Repeated electrical studies were performed. The EOG was normal in the left eye $(1.85)$ and borderline low in the right eye $(1 \cdot 66)$.

\section{CASE 8}

A 63-year-old white female, mother of case 7 , gave a history of blurred vision and poor night vision in both eyes since age 45 , the right eye being worse than the left. The symptoms had been stable for the past 3 to 4 years. Previous medical problems included Hashimoto's disease, which was treated medically with levothyroxine sodium $0.1 \mathrm{mg} / \mathrm{day}$. She

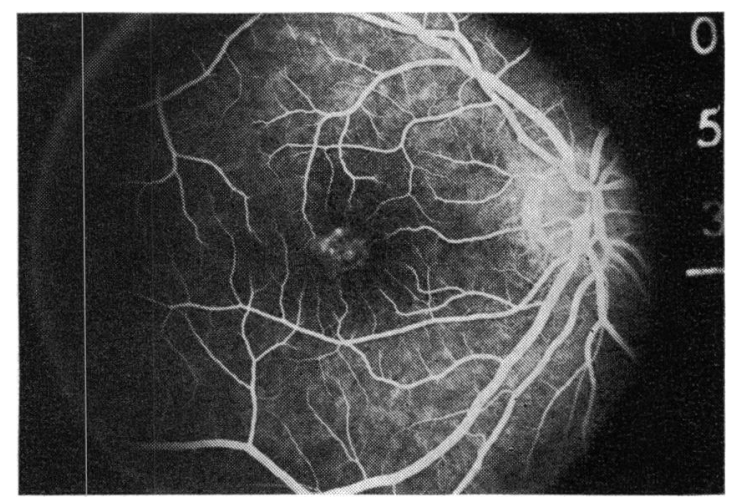

Fig. 10 (Case 7) Midvenous phase fluorescein angiogram with multiple RPE window defects in right macula. There was no leakage present in later photographs (1978). 
denied previous ocular disease or history of eye trauma.

The ocular examination revealed a best corrected vision of 20/80, Jaeger 4 in the right eye, and 20/40, Jaeger 2 in the left eye. Anterior segments, extraocular movements, pupils, and intraocular pressures were all within normal limits. Colour vision by isochromatic colour plates was normal bilaterally. Amsler grid studies showed distortion of the central 4 squares in the right eye. However, no field loss could be detected by Goldmann perimetry. Ophthalmoscopy revealed a $\frac{1}{4}$ to $1 / 3$ disc diameter, slightly elevated, vitelliform-like lesion in the right eye. The left eye appeared more consistent with a dry atrophic macular degenerative process.

Fluorescein angiography of the right eye disclosed an early hyperfluorescent ring type window defect in the macular region. Fluorescein angiography of the lesion in the left posterior pole revealed a similar window defect type of hyperfluorescence. The EOG was normal bilaterally, with light to dark trough ratios of 2.01 in the right eye and 1.90 in the left eye.

CASE 9

A 78-year-old woman had a gradual onset of blurred vision and distortion in both eyes since 1969. She had no refractive change or decrease in her visual status for the past 2 years. Ocular trauma or previous ocular disease was denied. Her medical problems included hypertension, atherosclerotic cardiovascular disease, and thyroid problems. Her medications included digoxin and a diuretic.

Visual acuity was 20/80 Jaeger 3 in the right eye, and 20/60, Jaeger 3 in the left eye. Pupils, extraocular movements, and intraocular pressures were all within normal limits. The lens showed early nuclear sclerotic changes in both eyes. Amsler grid, visual

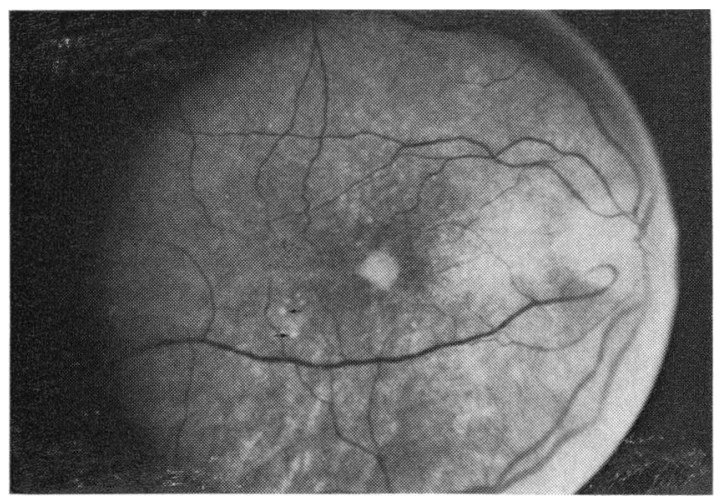

Fig. 11 (Case 9) Right eye adult vitelliform lesion in macula. Drusen are also present (1975). field, and colour vision were within normal limits bilaterally.

Examination of the 1975 photographs revealed bilateral $1 / 3$ disc diameter, vitelliform-like lesions in both eyes (Fig. 11). There was also diffuse retinal pigment epithelial thinning that exposed the underlying choroidal vasculature. Multiple punctate hypopigmented lesions at the level of the retinal pigment epithelium were seen in the left eye that were suggestive of drusen.

Fluorescein angiography in 1975 showed early hyperfluorescence in a doughnut type fashion that involved both macular regions. In addition small punctate lesions fluoresced in the midvenous phase, consistent with typical drusen. In the late angiographic stage there was a persistent window-defect type of hyperfluorescence in the macular region, with no evidence of pooling or leakage of dye.

Ophthalmoscopic examination in 1978 revealed multiple drusen-like changes in the left macular region that resembled atrophic macular degeneration. However, in the right eye, there was increased pigmentation in the macular region quite different from that in 1975 (Fig. 12). Fluorescein angiography similarly revealed early hyperfluorescence of both macular areas with evidence of perimacular drusen. Late fluorescein angiography revealed a ring-type window defect involving the right macular region. A similar pattern was seen in the left eye. No evidence of pooling or leakage could be seen. Electrical studies were not permitted.

\section{CASE 10}

A 63-year-old black female complained of blurred vision in both eyes since 1972. She denied previous ocular or medical disease, and family history was negative. Best corrected visual acuity was 20/30, Jaeger 2 in each eye. The anterior segments, pupils,

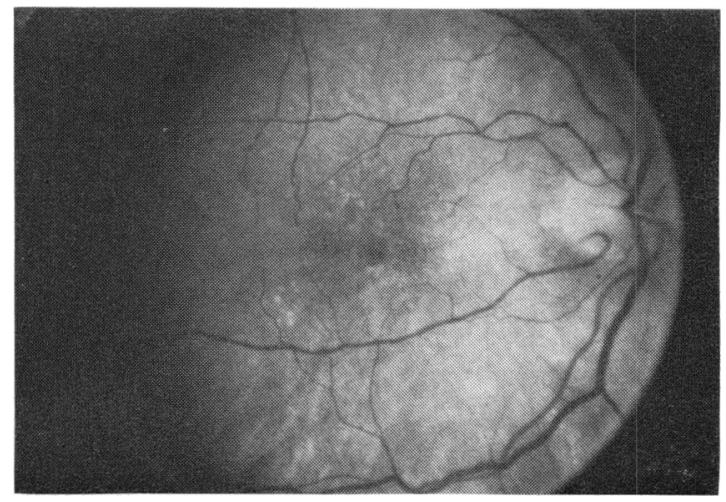

Fig. 12 (Case 9) 3 years later the vitelliform lesion has disappeared. Angiography was compatible with dry atrophic macular degeneration. 
extraocular movements, and intraocular pressures were normal bilaterally. Amsler grid, colour vision, and visual fields were normal in each eye. Ophthalmoscopy revealed bilateral, $1 / 3$ disc diameter vitelliform-like lesions. In addition there were scattered paracentral drusen. Angiography revealed hyperfluorescence in a window defect pattern similar to that seen in dry atrophic macular degeneration. Electrical studies were not permitted.

\section{Discussion}

The differential diagnosis of vitelliform or egg yolk lesions should include central serous choroidopathy, Best's disease, inflammatory aetiology (toxoplasmosis), retinal pigment epithelial detachment, macular drusen, and other macular degenerations (pseudovitelliform and peculiar foveal macular dystrophy). Our 10 patients were unusual in that the vitelliform-like lesions were atypical of Best's disease: (1) The lesions were smaller in size and with greater asymmetry. (2) Angiographically they did not block fluorescence. (3) The lesions occurred in an adult population. (4) EOG findings were normal. (5) The natural history differs.

Symptomatology in adult vitelliform macular degeneration consisted of decreased vision and metamorphopsia. The visual acuity was found to be symmetrical in most cases. In 3 cases $(1,6,7)$ a more hyperopic correction could improve the vision, which suggested a slight elevation of these macular lesions. In later stages of the disease there was little change in visual acuity in spite of the changing ophthalmoscopic and angiographic appearance. Acuity was generally better than 20/80, but may slowly decline with severe degenerative changes. Colour vision was found to be normal in most instances, though late stages of the disease showed a red-green anomaly.

One cannot predict the evolutional genetic patterns from our series of patients in spite of the examination of family members. However, there appeared to be a familial predisposition in 1 family (cases 7 and 8 ). We suggest that all family members of patients with vitelliform-like lesions are examined ophthalmoscopically.

Fluorescein angiographic patterns may vary. The most common angiographic finding in the initial presentation of this entity is perifoveal hyperfluorescence from pigment epithelial window defects, which is caused by focal pigment epithelial atrophy and dispersion. Pigment epithelial dispersion is commonly associated with clumping of $\mathrm{RPE}$. The foveolar region remains physiologically hypofluorescent. Fishman et al., in their cases of pseudovitelliform degeneration, indicated that peri- foveolar capillary leakage accounted for hyperfluorescence. ${ }^{13}$ Further follow-up and fluorescein angiogram of case 2 by stereo angiography revealed the leakage was subretinal rather than from the perifoveal capillary network. If retinal leakage of dye did indeed occur, one may expect a cystoid type configuration.

As the adult vitelliform lesion matures, an increase in subretinal exudation is noted. This accounts for the late subretinal leakage of dye presumably from the choriocapillaris. Subretinal haemorrhage or angiographic evidence of subretinal neovascularisation was not found in our cases. The natural history of the mature adult vitelliform lesion further varies. In 1 case (2) loss of RPE and choriocapillaris led to a clinical and angiographic picture of central areolar choroidal sclerosis. However, in 5 cases $(3,6,8,9$, and 10$)$ the adult vitelliform lesion disappeared and more closely resembled dry atrophic macular degeneration. Three cases $(5,9,10)$ had coexistent paracentral drusen.

These clinical and angiographic changes did not correleate significantly with changes in visual status. Fluorescein angiography should be utilised to differentiate other causes of vitelliform-like lesions as well as to ascertain the natural course.

Gass described in his series a central hyperpigmented spot in the fovea, presumed to be secondary to retinal pigment epithelial clumping. ${ }^{11} \mathrm{~A}$ similar finding was found in our cases $(1,2,3,4,7)$. Three of these patients did not show this finding in the initial examination. However, the central foveal hyperpigmentation did occur in the natural course of the disease on an average of 2 to 3 years later.

Electrical studies are useful in separating Best's disease from adult vitelliform macular degeneration. Krill found cases of retinal pigment epithelium and sensory retinal detachment having normal to slightly subnormal EOGs..$^{15}$ In senile macular degeneration and dominant drusen he found normal EOGs. The findings in our patients were consistent with those found in dry atrophic macular degeneration. An extinguished or markedly subnormal EOG, which is found in Best's disease, was not found in our cases. For those patients in whom a familial tendency is a consideration electrical studies further delineate true vitelliform dystrophy from adult vitelliform macular degeneration (see Table 1).

Without histopathological studies the location and the natural course of events cannot be proved. The initial degenerative process may be in the retinal pigment epithelium or choriocapillaris. Gass found histological evidence of depigmentation, degeneration, and disruption of retinal pigment epithelium centrally. ${ }^{11}$ Also, periodic-acid-Schiff- 
Table 1 Differentiation of adult vitelliform macular degeneration from vitelliform macular dystrophy

\begin{tabular}{|c|c|c|}
\hline & $\begin{array}{l}\text { Vitelliform } \\
\text { dystrophy }\end{array}$ & $\begin{array}{l}\text { Adult vitelliform } \\
\text { degeneration }\end{array}$ \\
\hline Age on onset & Prior to 2 nd decade & $\begin{array}{l}\text { Greater than } 40 \text { years } \\
\text { of age }\end{array}$ \\
\hline Size of lesion & $\frac{1}{2}-3$ disc diameters & $1 / 3-1$ disc diameters \\
\hline Symmetry & $+t$ & $+t+$ \\
\hline \multirow[t]{2}{*}{ Visual acuity } & $\begin{array}{l}\text { Initially normal or } \\
\text { slightly subnormal }\end{array}$ & Initially $20 / 30-20 / 80$ \\
\hline & $\begin{array}{l}\text { Variable decline } \\
\text { eventual }\end{array}$ & $\begin{array}{l}\text { Slow progression, } \\
\text { vision visually stable }\end{array}$ \\
\hline Colour vision & $\begin{array}{l}\text { Red-green anomally } \\
\text { in late stages }\end{array}$ & $\begin{array}{l}\text { Red-green anomally } \\
\text { in late stages }\end{array}$ \\
\hline $\begin{array}{l}\text { Fluorescein } \\
\text { angiography }\end{array}$ & $\begin{array}{l}\text { Vitelliform lesion } \\
\text { hypofluorescent }\end{array}$ & $\begin{array}{l}\text { Vitelliform lesion with } \\
\text { ring-like RPE } \\
\text { window defect }\end{array}$ \\
\hline EOG & Subnormal & Normal \\
\hline Genetic aspects & Autosomal dominant & No definitive pattern \\
\hline
\end{tabular}

positive subpigment epithelial deposition of eosinophilic material was seen paracentrally. We believe that the primary pathological process in adult vitelliform macular degeneration is at the level of the retinal pigment epithelium as shown by fluorescein angiography. However, as the disease progresses, there may be additional degeneration of the choriocapillaris.

The natural progression of the disease shows that there is a change in the clinical as well as angiographic findings in most patients, especially in the later years of life. The visual acuity, however, usually did not markedly decrease. The eventual clinical picture in 5 cases was indistinguishable from that of dry atrophic macular degeneration. Three patients had coexistent paracentral typical drusen. It is our belief that adult vitelliform macular degeneration is a form of dry atrophic macular degeneration. The adult vitelliform lesion may be equal to macular drusen in forecasting the eventual clinical picture and natural history of dry atrophic macular degeneration. However, none of our cases showed exudative macular degenerative changes in spite of being followed up for periods of more than 5 years.

We thank Mr Tom Quirk and Mr Burt Green for the exceptional photography. We acknowledge Frank La Franco, MD, and Andrew Lewicky. MD, for their technical assistance. Thanks also to Debbi Campbell for typing the manuscript.

\section{References}

${ }^{1}$ Best F. Ueber eine hereditare Maculoaffektion. Beitrage zur Vererbunglehre. $Z$ Augenheilkd 1905; 13: 199-212.

${ }^{2} Z$ anen. Rausen-kyste vitelliform congénital de la macula. Bull Soc Belge Ophtalmol 1950; 96: 544-9.

${ }^{3}$ Krill AE, Morse PA, Potts AM, Klien BA. Hereditary vitelliruptive macular degeneration. Am J Ophthalmol 1968; 61 : 1405-114.

${ }^{4}$ Braley AE, Spivey BE. Hereditary vitelline macular degeneration. Arch Ophthalmol 1964; 72: 743-62.

${ }^{5}$ Deutman AF. Electro oculography in families with vitelliform dystrophy of the fovea. Arch Ophthalmol 1969; 81: 305-16.

${ }^{6}$ Deutman AF. Hereditary Dystrophies of the Posterior Pole of the Eye. Assen: Van Gorcum, 1971.

'Sorby A, Savory M, Davey JB, Fraser RJL. Macular cysts; A dominantly inherited affection with a progressive course. Am J Ophthalmol 1956; 40: 144-58.

${ }^{8}$ Goldberg MF. Genetic and Metabolic Eye Disease. Boston: Little, Brown, 1974.

${ }^{9}$ Morse PH, Maclean AL. Fluorescein fundus studies in hereditary vitelliruptive macular degeneration. $\mathrm{Am} J$ Ophthalmol 1968; 66: 485-94.

${ }^{10}$ Curry HF; Moorman LT. Fluorescein photography of vitelliform macular degeneration. Arch Ophthalmol 1968; 79: 705-9.

${ }^{11}$ Gass JDM. A clinicopathologic study of a peculiar foveomacular dystrophy. Trans Am Ophthalmol Soc 1974; 72: 139-56.

${ }^{12}$ Gass JDM. Stereoscopic Atlas of Macular Diseases, 2nd ed. St Louis: Mosby, 1977.

${ }^{13}$ Fishman GA, Trimble S, Rabb MF, Fishman M. Pseudovitelliform macular degeneration. Arch Ophthalmol 1977; 95: 73-6.

${ }^{14}$ Kingham JO, Lochen GP. Vitelliform macular degeneration. Am J Ophthalmol 1977; 84: 531-6.

${ }^{15} \mathrm{Krill}$ AE. The electroretinographic and electro oculographic findings in patients with macular lesions. Trans Am Acad Ophthalmol Otolaryngol 1966; 70: 1063-83. 